\title{
BALANCED BINARY ARRAYS III: THE HEXAGONAL GRID
}

\author{
SHEILA OATES-WILLIAMS and ANNE PENFOLD STREET
}

(Received 29 June 1979)

Communicated by W. D. Wallis

\begin{abstract}
We consider the following problem arising in agricultural statistics. Suppose that a large number of plants are set out on a regular grid, which may be triangular, square or hexagonal, and that among these plants, half are to be given one and half the other of two possible treatments. For the sake of statistical balance, we require also that, if one plant in every $k$ plants has $i$ of its immediate neighbours receiving the same treatment as itself, then $k$ is constant over all possible values of $i$. For square and triangular grids, there exist balanced arrays of finite period in each direction, but for the hexagonal grid, we show that no such balanced array can exist. Several related questions are discussed.
\end{abstract}

1980 Mathematics subject classification (Amer. Math. Soc.): 05 B 30, 05 B 45, 62 K 99.

\section{Introduction}

We continue in this paper our earlier work (Street and Macdonald (1979), Macdonald and Street (1979)) concerning the following problem. Plants grown in large numbers for commercial purposes are usually set out on a regular grid, triangular, square or hexagonal. In order to space them for optimal yield, it is necessary to study the way they behave when competing with each other for light.

Consider the experimental arrangements in a greenhouse. Over each plant there is a lamp; half the plants are to have their lamps on and half off. The intensity of light falling on any particular plant is determined firstly by whether its own lamp is on and secondly by the number of its immediate neighbours whose lamps are on. (We may ignore more distant lamps to within a reasonable approximation.)

For example, in the hexagonal grid, each plant has six immediate neighbours. Thus each plant can lie at the centre of any of $2^{6}=64$ possible neighbourhoods, 

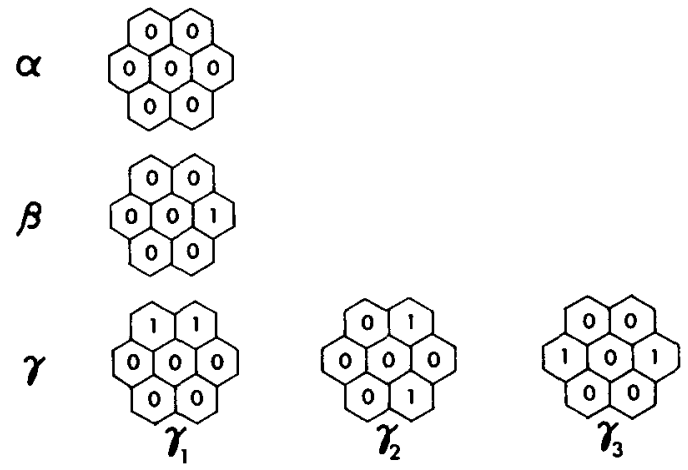

$6 \underbrace{011}_{\delta_{1}}$
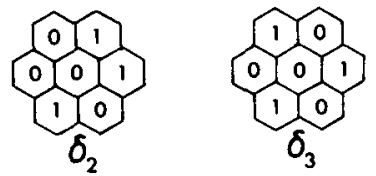

\&
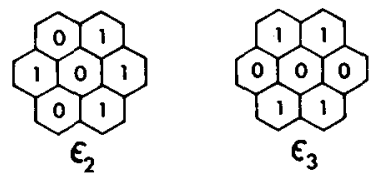

$5 \underbrace{100}_{100}$

$\eta$ 1011

FIGURE 1. The equivalence classes of the neighbourhoods of 0 .

TABLE 1

The distribution of the neighbourhoods of 0 into equivalence classes

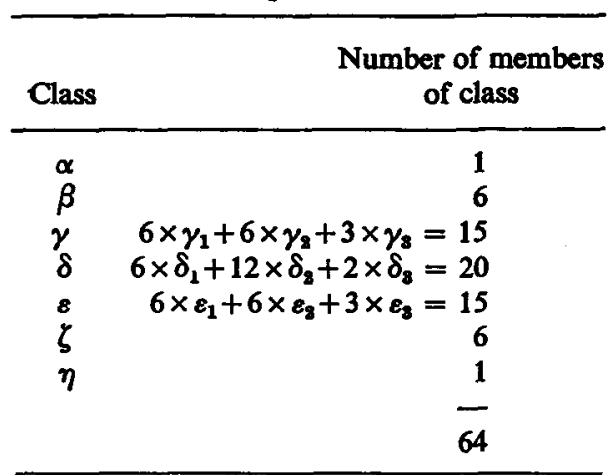


and these 64 neighbourhoods fall into seven equivalence classes, depending on how many of the neighbouring plants have their lamps in the same state as the central lamp.

Suppose that 0 denotes a plant whose lamp is on, and 1 a plant whose lamp is off. Figure 1 shows representatives of the seven equivalence classes of neighbourhoods of 0 ; note that three of the seven classes split up naturally into three subclasses each. Table 1 shows the number of members of each class and subclass. We always label the configuration according to the number of neighbours which match the central element; thus 1 is in the $\alpha$ configuration when surrounded by 1 s.

For the sake of statistical balance, the array is required to consist of equal numbers of 0 s and $1 \mathrm{~s}$ and, further, to have equal numbers of each symbol in each configuration. Hence on average, of every fourteen symbols in the array, we should have seven 0 s and seven $1 \mathrm{~s}$, and of the seven symbols of each type, one should be in each of the $\alpha, \beta, \gamma, \delta, \varepsilon, \zeta$ and $\eta$ configurations.

Previously we constructed periodic balanced arrays for the square and triangular grids respectively, and showed that these arrays could satisfy various additional conditions which simplified their applications. By contrast for the hexagonal grid, we show in Section 2 that no such periodic balanced array can exist. However, there do exist binary hexagonal arrays with various interesting properties and in Section 3 we discuss some of these arrays. Some unsolved problems are listed in Section 4.

\section{The non-existence result}

Our main result is the following

THEOREM. For the hexagonal grid, no periodic balanced binary array can exist.

Proof. We proceed by a counting argument, showing that it is impossible to fit enough $\zeta s$ and $\eta$ s into the array. Since the proof depends strongly on the geometry of the grid, we use numerous diagrams and for clarity we let white and dotted hexagons correspond to 0 s and $1 \mathrm{~s}$ respectively throughout.

By definition, a white hexagon cannot border another white hexagon in the $\eta$ configuration. From Figure 1, we see that a white hexagon in the $\alpha$ or $\eta$ configuration cannot border a dotted $\eta$ nor a white nor dotted $\zeta$. However, a white $\beta$ can border a dotted $\zeta$ or a dotted $\eta$, though never a white $\zeta$, and so on. Hence with each white hexagon in an array, we may associate a vector $(a, b, c)$ of non-negative integer components, such that $a, b$ and $c$ are the number of white $\zeta$ s, dotted $\zeta$ s and dotted $\eta s$ respectively, bordered by the original white hexagon. 
We say that the vector $\left(a_{1}, b_{1}, c_{1}\right)$ covers the vector $\left(a_{2}, b_{2}, c_{2}\right)$ if and only if $a_{1} \geqslant a_{2}, b_{1} \geqslant b_{2}$ and $c_{1} \geqslant c_{2}$. Then for each configuration of a white hexagon, we must have $a$ covering family of vectors, that is, vectors which can be associated with the given configuration and such that any vector associated with the configuration is covered by at least one vector of the family. Table 2 shows the covering vectors for each configuration of the white hexagons and Figure 2 shows the associated neighbourhoods.

TABLE 2

Covering families of vectors and sums of their components

\begin{tabular}{|c|c|c|c|c|}
\hline White hexagon & $\begin{array}{l}\text { Bordering } \\
a \text { white } \zeta \mathrm{s}\end{array}$ & $\begin{array}{l}\text { Bordering } \\
b \text { dotted } \zeta \text { s }\end{array}$ & $\begin{array}{l}\text { Bordering } \\
c \text { dotted } \eta s\end{array}$ & $a+b+c$ \\
\hline$\alpha$ & 0 & 0 & 0 & 0 \\
\hline \multirow[t]{2}{*}{$\beta$} & $\mathbf{0}$ & 0 & 1 & 1 \\
\hline & $\mathbf{0}$ & 1 & 0 & 1 \\
\hline$\gamma_{1}$ & $\mathbf{0}$ & 2 & 0 & 2 \\
\hline \multirow[t]{3}{*}{$\gamma_{2}$} & 1 & 2 & 0 & 3 \\
\hline & 0 & 0 & 2 & 2 \\
\hline & 0 & 1 & 1 & 2 \\
\hline \multirow[t]{3}{*}{$\gamma_{3}$} & 0 & 2 & $\mathbf{0}$ & 2 \\
\hline & $\mathbf{0}$ & 1 & 1 & 2 \\
\hline & $\mathbf{0}$ & 0 & 2 & 2 \\
\hline$\delta_{1}$ & 0 & 2 & $\mathbf{0}$ & 2 \\
\hline \multirow[t]{3}{*}{$\delta_{2}$} & 0 & 3 & 0 & 3 \\
\hline & $\mathbf{0}$ & 2 & 1 & 3 \\
\hline & 1 & 2 & $\mathbf{0}$ & 3 \\
\hline \multirow[t]{5}{*}{$\delta_{8}$} & $\mathbf{0}$ & 0 & 3 & 3 \\
\hline & $\mathbf{0}$ & 1 & 2 & 3 \\
\hline & 3 & 0 & $\mathbf{0}$ & 3 \\
\hline & 1 & 2 & 1 & 4 \\
\hline & 1 & 3 & $\mathbf{0}$ & 4 \\
\hline$e_{1}$ & 0 & 2 & 0 & 2 \\
\hline \multirow[t]{4}{*}{$\varepsilon_{2}$} & 2 & 0 & $\mathbf{0}$ & 2 \\
\hline & 1 & 2 & 0 & 3 \\
\hline & 0 & 3 & $\mathbf{0}$ & 3 \\
\hline & 0 & 2 & 1 & 3 \\
\hline \multirow[t]{3}{*}{$\varepsilon_{3}$} & 0 & 4 & 0 & 4 \\
\hline & 1 & 2 & 0 & 3 \\
\hline & 2 & 0 & $\mathbf{0}$ & 2 \\
\hline \multirow[t]{2}{*}{$\zeta$} & 0 & 2 & 0 & 2 \\
\hline & 1 & 0 & 0 & 1 \\
\hline$\eta$ & 0 & 0 & 0 & 0 \\
\hline
\end{tabular}

Suppose that a periodic balanced binary array does in fact exist. Then it contains a block of $14 n$ hexagons, $7 n$ white and $7 n$ dotted, with $n$ of each colour in each configuration, that is, a balanced block for some integer $n$. From Table 2, we see 
that a white hexagon may border at most three dotted $\eta \mathrm{s}$, at most three white $\zeta$ s, at most four dotted $\zeta s$ and a total of at most four of these three types of hexagons. Let $k_{i}, k_{i}^{\prime}$ and $l_{i}$ denote the number of white hexagons in the balanced block which border $i$ dotted $\zeta$ s, $i$ white $\zeta$ s and $i$ dotted $\eta \mathrm{s}$, respectively. Similarly let $m_{i}$ denote the number which border a total of $i$ dotted $\zeta$ s, white $\zeta$ s and dotted $\eta s$. Now each dotted $\zeta$ has five of its six edges adjacent to white hexagons and from Table 2 we see that $k_{\mathrm{b}}=k_{\mathrm{B}}=0$, so that

$$
k_{1}+2 k_{2}+3 k_{3}+4 k_{4}=5 n .
$$

Similarly, counting edges of the white $\zeta$ s, we have

$$
k_{1}^{\prime}+2 k_{2}^{\prime}+3 k_{3}^{\prime}=n
$$

for the dotted $\eta s$, we have

$$
l_{1}+2 l_{2}+3 l_{3}=6 n
$$

and for all of them together

$$
m_{1}+2 m_{2}+3 m_{3}+4 m_{4}=12 n .
$$

Suppose that of the $n$ white hexagons in each configuration in the balanced block, we have $n \lambda \varepsilon_{3}$ s contributing to $m_{4}$, that is $n \lambda \varepsilon_{3}$ s of type $(0,4,0), n \mu \delta_{3} \mathrm{~S}$ contributing to $m_{4}$, that is of types $(1,2,1)$ or $(1,3,0)$, and $n \nu \gamma_{2}$ s contributing to $m_{3}$, that is of type $(1,2,0)$, where $0 \leqslant \lambda, \mu, \nu \leqslant 1$.

Notice (see Figure 2) that each white $\varepsilon_{3}$ of type $(0,4,0)$ has its two white neighbours in configurations $\gamma_{2}$ or $\delta_{3}$, and, moreover, the white neighbours of distinct $\varepsilon_{3} \mathrm{~s}$ of this type are distinct. The $\gamma_{2} \mathrm{~s}$ are of type $(0,2,0)$ (covered by $(1,2,0)$ ) and the $\delta_{3}$ s of type $(0,2,1)$ (covered by $\left.(1,2,1)\right),(0,3,0)$ (covered by $(1,3,0)$ ) or $(0,2,0)$ (covered by either). In particular, the $\gamma_{2} \mathrm{~s}$ contribute to no $l_{i}$, and the $\delta_{3} \mathrm{~s}$ at most to $l_{1}$. Let there be $n \lambda_{1}$ such $\gamma_{2}$ s and $n \lambda_{2}$ such $\delta_{3}$ s, so that

$$
\lambda_{1}+\lambda_{2}=2 \lambda
$$

Also each $\delta_{3}$ contributing to $m_{4}$ is of type $(1,2,1)$ or $(1,3,0)$ and contributes at most to $l_{1}$, and each $\gamma_{2}$ contributing to $m_{3}$ is of type $(1,2,0)$ and contributes to no $l_{i}$.

Now consider the white hexagons bordering the dotted $\eta \mathrm{s}$. A contribution to $l_{3}$ could arise only from $\delta_{3}$ s not contributing to $m_{4}$, nor bordering $\varepsilon_{3} s$ of type $(0,4,0)$. A contribution to $l_{2}$ could arise only from $\gamma_{3} \mathrm{~s}$ or $\gamma_{2} \mathrm{~s}$ not contributing to $m_{3}$, nor bordering $\varepsilon_{3} s$ of type $(0,4,0)$ (or, of course, from the $\delta$ s just mentioned, but this would decrease the total contribution). Finally, a contribution to $l_{1}$ can then arise 

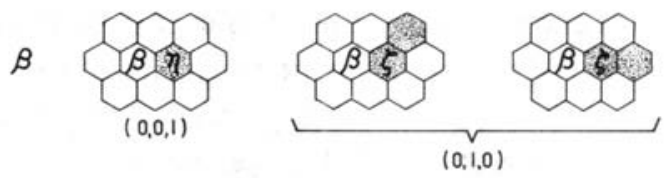

$\boldsymbol{\gamma}_{1}$

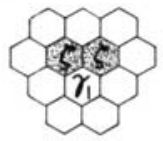

$(0,2,0)$
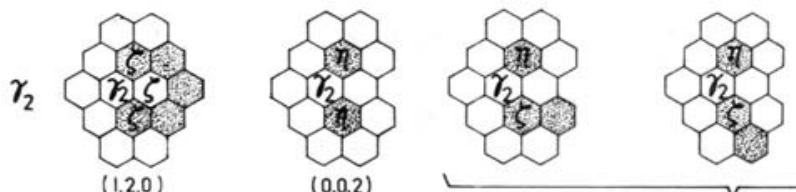

$(0,1,1)$

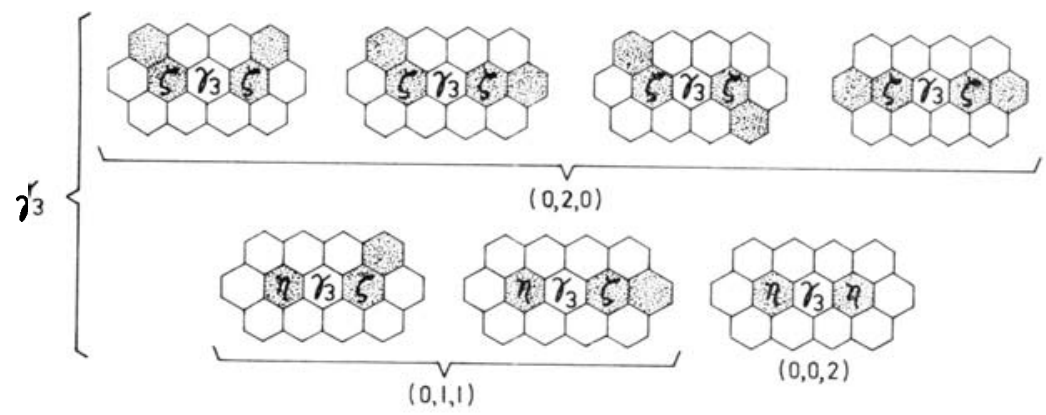

$\delta_{1}$

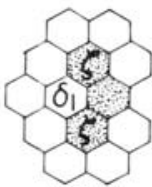

$(0,2,0)$

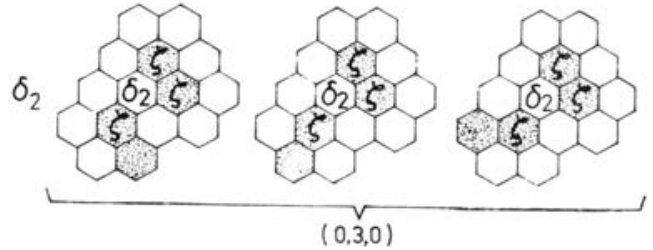

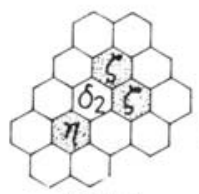

$(0,2,1)$

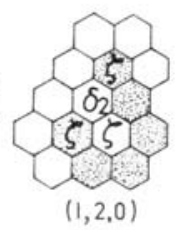

$(1,2,0)$

Frgure 2. Configurations of white hexagons associated with covering families of vectors. 

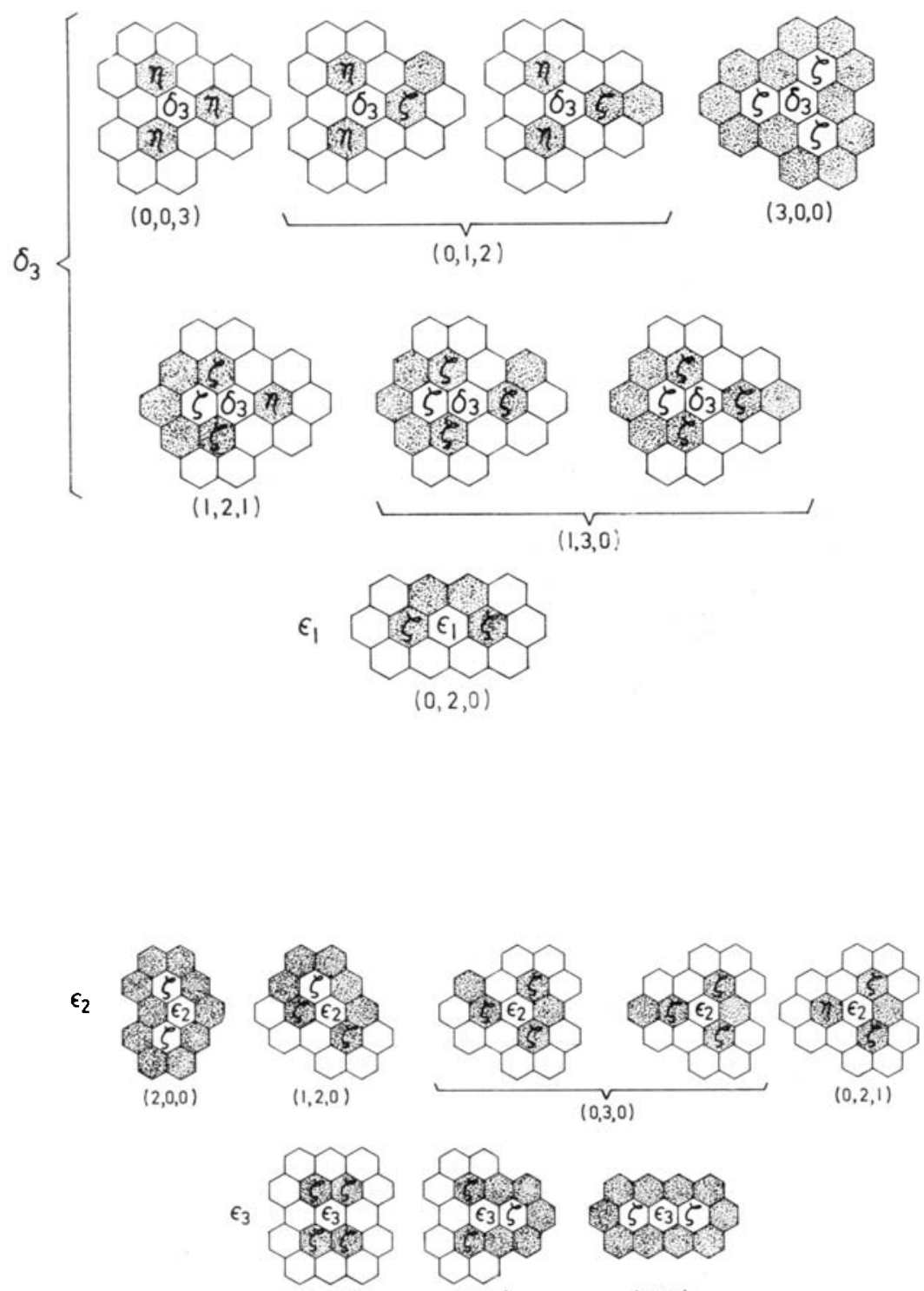

$(0,4,0)$

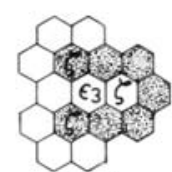

$(1,2,0)$

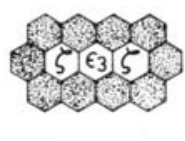

$(2,0,0)$

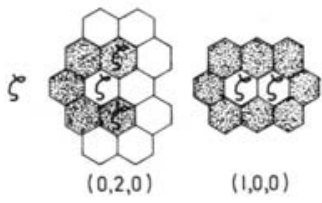

Figure 2 (cont.) 
from $\beta$ s, from $\varepsilon_{2} s$ and from $\delta_{3}$ s contributing to $m_{4}$ or bordering $\varepsilon_{3} s$ of type $(0,4,0)$. Hence from (3)

$$
\begin{aligned}
6 n & =l_{1}+2 l_{2}+3 l_{3} \\
& \leqslant n\left[1+\left(\lambda_{2}+\mu\right)+(1-\lambda)\right]+2 n\left(1-\lambda_{1}-\nu\right)+3 n\left(1-\lambda_{2}-\mu\right) \\
& =n\left[7-2\left(\lambda_{1}+\lambda_{2}\right)-\lambda-2 \nu-2 \mu\right] \\
& =n[7-(5 \lambda+2 \nu+2 \mu)]
\end{aligned}
$$

by (5), so that

$$
5 \lambda+2 \nu+2 \mu \leqslant 1
$$

We deal similarly with equation (4). Contributions to $m_{4}$ come from the $n \lambda \varepsilon_{3} \mathrm{~s}$ and the $n \mu \delta_{3}$ s only. Contributions to $m_{3}$ can come from the remaining $\varepsilon S$ and $\delta s$ and from the $n \nu \gamma_{2}$ s. Contributions to $m_{2}$ can come from the $\zeta \mathrm{s}$ and the remaining $\gamma \mathrm{s}$, and to $m_{1}$ from the $\beta$ s. (Again we could, for instance, have a $\zeta$ contributing only to $m_{1}$, but this would decrease the total contribution.) Hence by (4),

$$
\begin{aligned}
12 n & =m_{1}+2 m_{2}+3 m_{3}+4 m_{4} \\
& \leqslant n+2 n[1+(1-\nu)]+3 n[(1-\lambda)+(1-\mu)+\nu]+4 n(\lambda+\mu) \\
& =n(11+\lambda+\mu+\nu) \\
& \leqslant 11 \frac{1}{2} n
\end{aligned}
$$

by (6), which is a contradiction.

So no such periodic balanced binary array can exist.

Figure 3 shows a double-strip pattern, of period 18 along the strip, which contains, for every 9 hexagons of either colour, one each of $\alpha, \beta, \gamma, \zeta$ and $\eta$ and two each of $\delta$ and $\varepsilon$. This array is the closest to being balanced that we have so far constructed.

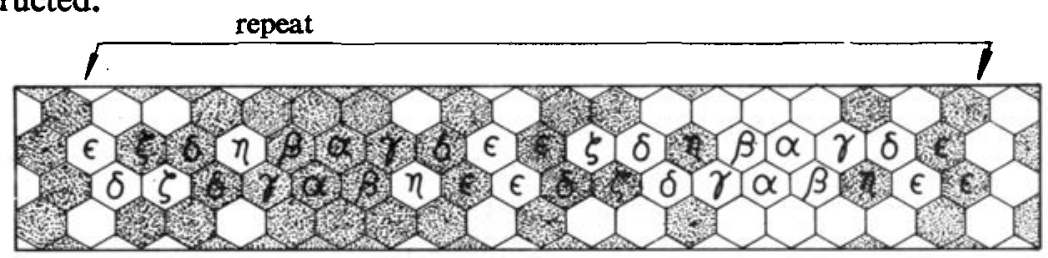

FIGURE 3. Strip pattern.

We point out in this connection that each of the balanced tilings we constructed for the square and triangular grids were associated with uniform tilings of the plane not made up of edge-to-edge tiles: see Street and Macdonald (1979), Figures 
28 and 30, and Macdonald and Street (1979), Figures 15 and 16. However, none of the eight such families of tilings can be built up from regular hexagons; see Grünbaum and Shephard (1977). But we have been unable to use this observation to simplify the proof of the Theorem.

\section{Periodic binary arrays}

In the square and triangular grids, the balanced arrays of special interest are those we called sequential, that is, those which have the same periodic binary sequence repeated along every row of the array, in every direction. They are particularly suitable for application and we therefore began to look for sequential arrays in the hexagonal grid, hoping to find some which, like the non-sequential array of Figure 3, approached reasonably close to balance.

It is easy to check that no sequential array of period two can exist, so the shortest possible period is three, with sequence 011 as in Figure 4.

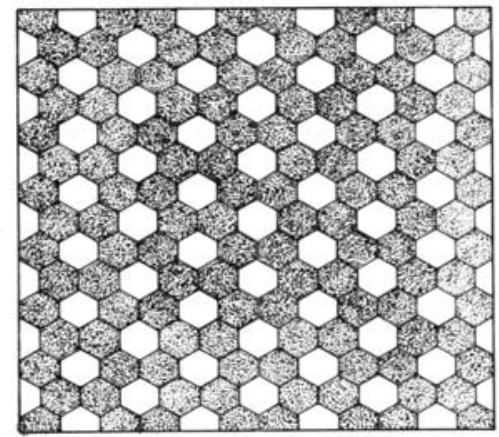

FIOURE 4. Sequential array with sequence 011.

This simple array is of interest for several reasons.

LEMMA 1. For the hexagonal grid, the sequential array of Figure 4 is the only one in which one of the symbols occurs only in 2-strings.

Proof. Without loss of generality, suppose that 1 occurs only in 2-strings. Consider the array shown in Figure 5. Since 1 occurs only in 2-strings, we must have the subsequence 0110 . If position 3 is occupied by a 1 , then all positions labelled 2 must be occupied by 0 s. If $X$ is occupied by a 1 , then it is an isolated 1 in the sequence in the one o'clock direction; hence $X$ is filled by a 0 . Similarly, $Y$ must be filled by 0 , to avoid an isolated 1 in the sequence in the eleven o'clock direction. But now the 1 in position 3 is isolated in the horizontal direction and we have a contradiction. Hence the hexagons immediately above and below the 2-string of $1 \mathrm{~s}$ must be occupied by 0 s, and we are led back to the array of Figure 4. 


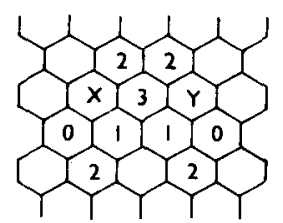

FIGURE 5.

LEMMA 2. For the hexagonal grid, no sequential array can exist in which one of the symbols occurs only in 3-strings.

Proof. Suppose that such an array did exist, with 1 occurring only in 3-strings and consider the cases shown in Figure 6. We begin with the array of Figure 6(a) and consider the symbols in positions $X$ and $Y$. If both $X$ and $Y$ are occupied by 0 s, we have Figure 6(b). All the hexagons labelled 3 must be filled by $1 \mathrm{~s}$, all those labelled 2 by 0 s. The row marked with an arrow now contains a 4-string of $1 \mathrm{~s}$, which is impossible.

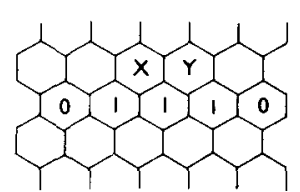

(a)

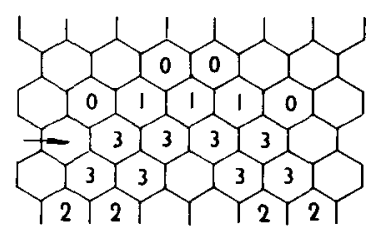

(b)

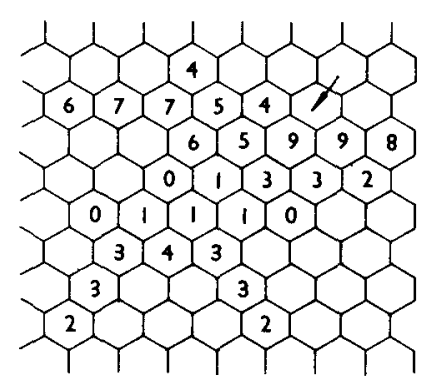

(c)

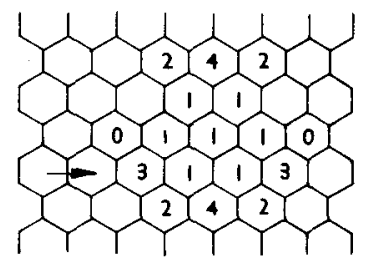

(d)

FIGURE 6.

If $X=0$ and $Y=1$, we have Figure 6(c). Now all the hexagons labelled 3 are filled by $1 \mathrm{~s}$ and those labelled 2 by 0 s. This in turn forces hexagons labelled 5 (and later 7 and 9 ) to be filled by $1 \mathrm{~s}$, and those labelled 4 (and later 6 and 8) by 0 s, leading to the marked 4-string of $1 \mathrm{~s}$, again a contradiction.

We may now assume that the four hexagons immediately above and below the 3-string of $1 \mathrm{~s}$ are occupied by $1 \mathrm{~s}$, as in Figure 6(d). This forces hexagons labelled 2 (and 4 ) to be filled by 0 s, and those labelled 3 by $1 \mathrm{~s}$, giving a forbidden 4 -string as before. 
Lemmas 1 and 2 between them rule out the possibility of a sequential array of period 4. However, arrays of all odd periods do exist, with sequences $0 \underbrace{011 \ldots 1}_{2 n}$; thesa may be regarded as members of a family beginning with the array of Figure 4 . They are shown for $n=2$ and 6 in Figures 7(a) and (b) respectively.

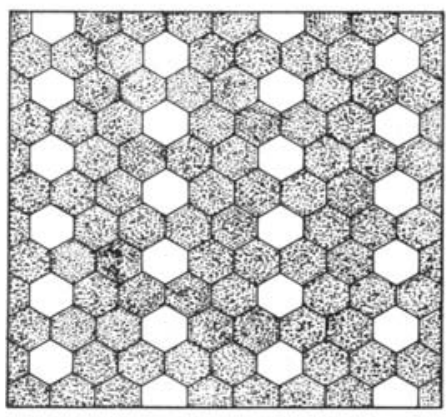

(a)

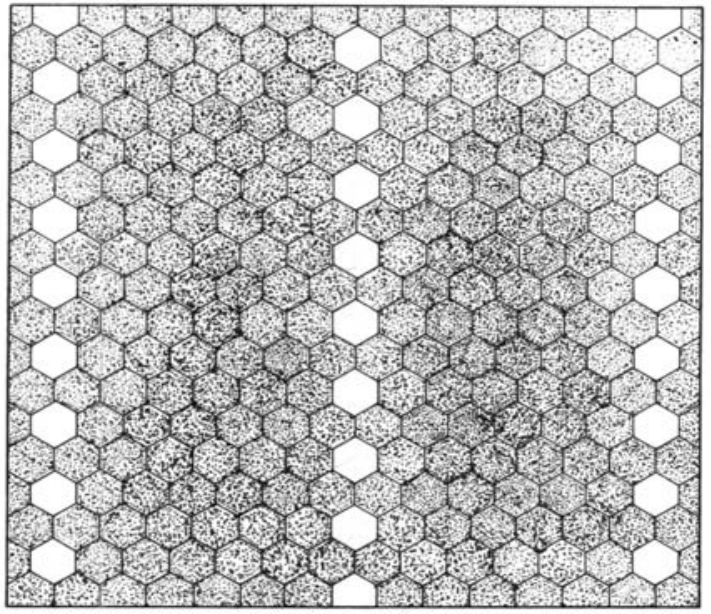

(b)

FIGURE 7. Sequential arrays with two-fold symmetry:

(a) sequence 01111 ; (b) sequence 0111111111111 .

For $n=2$, the sequence 01111 can easily be shown to give the unique array of Figure 7(a). For larger values of $n$, the array is not necessarily determined uniquely by the sequence as, for example, the array of Figure 8 has the same sequence as

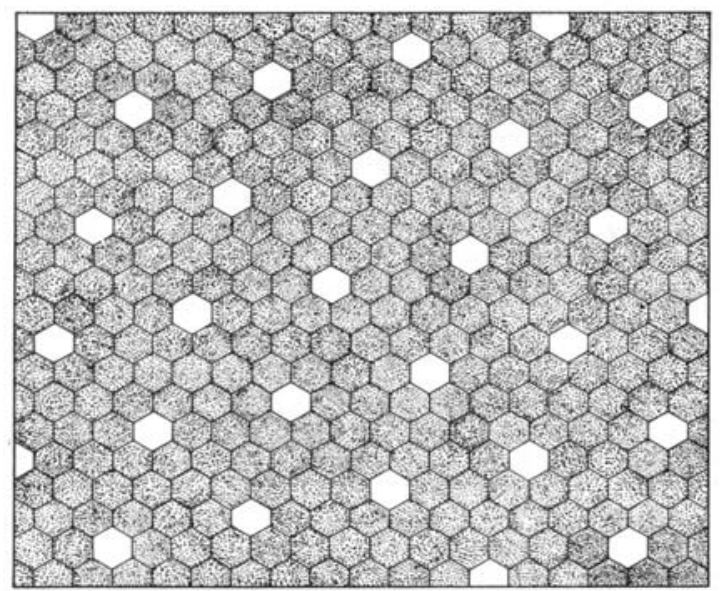

FIGURE 8. Sequential array with six-fold symmetry: sequence 0111111111111 as for Figure 7(b). 


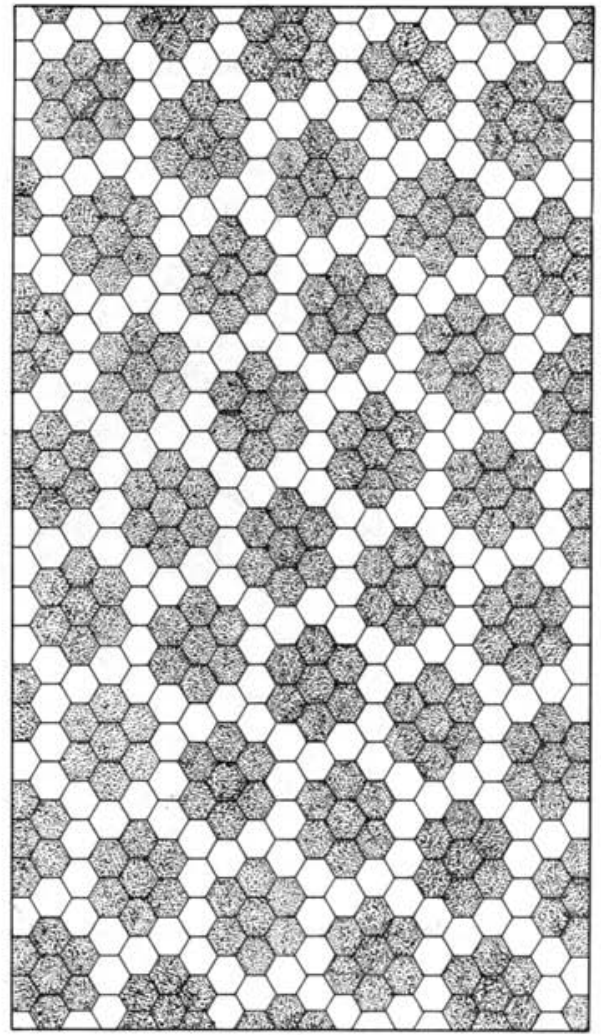

(a)

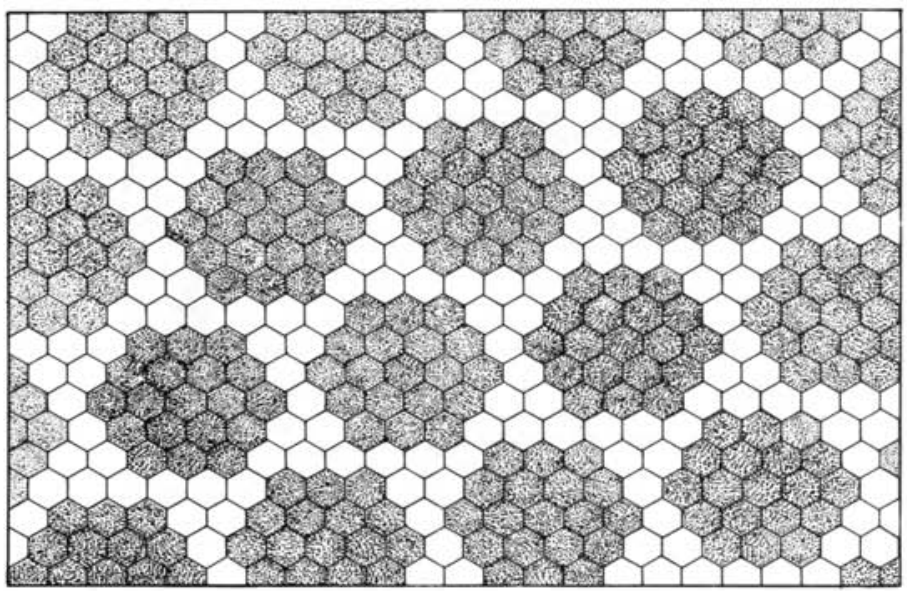

(b)

FIGURE 9. Sequential arrays of period $f(n)=4 n^{2}-2 n+1$ : (a) six-fold symmetry, period 13, $n=2$; (b) six-fold symmetry, period $31, n=3$. 
that of Figure 7(b). Notice also that the array of Figure 7(b) has two-fold symmetry, whereas that of Figure 8 has six-fold symmetry, so that the sequence does not even determine the symmetry properties of the array.

The array of Figure 4 may also be regarded as the smallest member of another family, several members of which are shown in Figure 9. If the dotted hexagonal motif of the array has side $n$, then it contains $1+3 n(n-1)$ small hexagons; the entire sequence runs also through the equivalent of the two white triangular motifs, each of side $n$, containing between them $m(m+1)$ small hexagons. Thus the sequence has period $f(n)=1+3 n(n-1)+n(n+1)=4 n^{2}-2 n+1$, for each positive integer $n$, and for each value of $f(n)$, such a sequential array exists.

Another family of sequential arrays with six-fold symmetry are the snowflake arrays, two of which are shown in Figure 10. If $n$ denotes the number of small shaded hexagons to the left of the white centre of the snowflake, then each snowflake contains $6 n(n+1)+1$ small hexagons, and by allowing for the $3 n(n+1)+2$ small hexagons lying between the snowflakes, we see that the periods of these arrays are given by

$$
\begin{aligned}
g(n) & =6 n(n+1)+1+3 n(n+1)+2 \\
& =3\left(3 n^{2}+3 n+1\right) .
\end{aligned}
$$

Figure 11 shows a sequential array of cog-wheels, again with six-fold symmetry, of interest because its sequence contains only isolates and 2-strings of one symbol (0) and only isolates, 2- and 3-strings of the other (1). The sequence has length 43, namely

\section{1.}

Many asymmetric motifs may be arranged to give sequential arrays with six-fold symmetry. An example of period 91 is shown in Figure 12.

Arrays $P$ and $Q$ of co-prime periods $p$ and $q$, say, at least one of which has sixfold symmetry, may be composed to give a new array $P Q$ of period $p q$. To compose the arrays, suppose that $P$ has six-fold symmetry and contains non-overlapping motifs with six-fold symmetry. Choose two colouring schemes (with six-fold symmetry) for the motifs of $P$, and colour them in accord with the arrangement of $Q$. For example, to compose the arrays $P$ of Figure 9(a) and $Q$ of Figure 4, we choose the colouring schemes shown in Figure 13 for the motifs of $P$. We now colour the motifs of $P$ according to the array $Q$, that is, ...abbabb ... along each axis of symmetry of the array $P$. Figure 14(a) shows the composition in this order, and Figure 14(b) the composition with $P$ and $Q$ interchanged.

All the arrays discussed so far have had either two-fold or six-fold symmetry. Consequently their sequences read the same in either direction. But there also exist arrays with three-fold symmetry for which this is not true, for example, the arrays in Figure 15, which are derived in an obvious way from those of Figure 9 


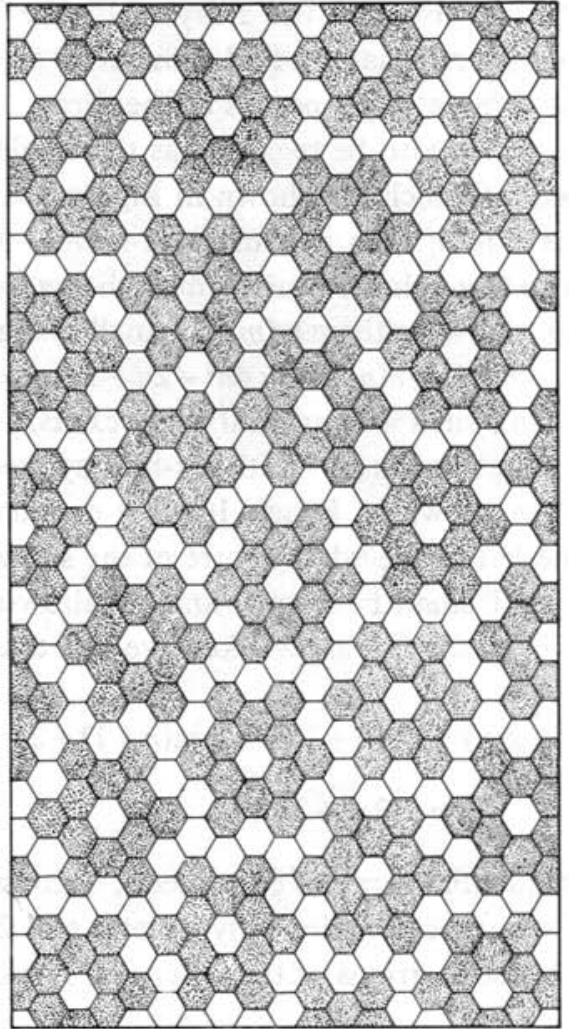

(a)

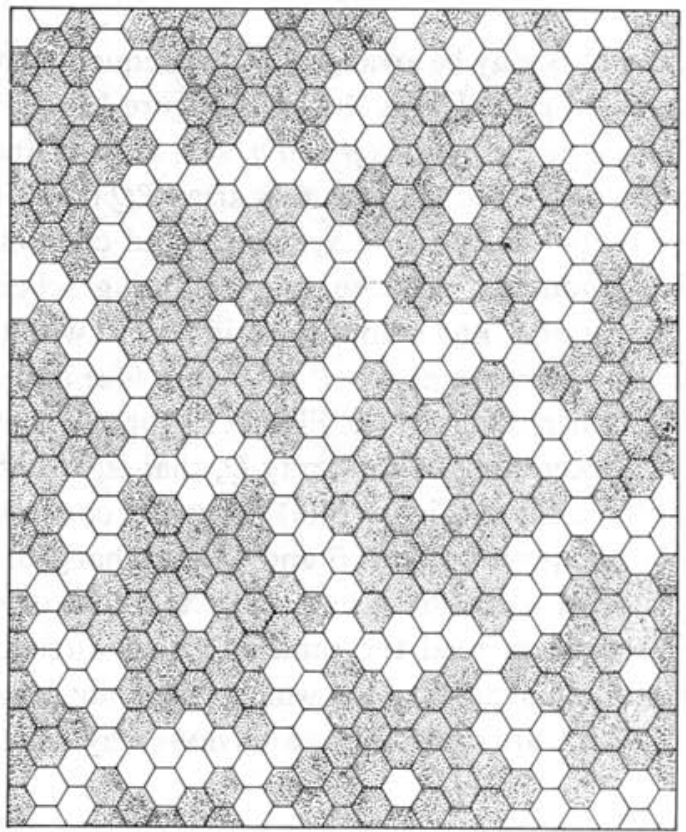

(b)

FIGURE 10. Snowflake arrays, sequential of period $g(n)=3\left(3 n^{2}+3 n+1\right)$ : (a) period $21, n=1$; (b) period $57, n=2$. 


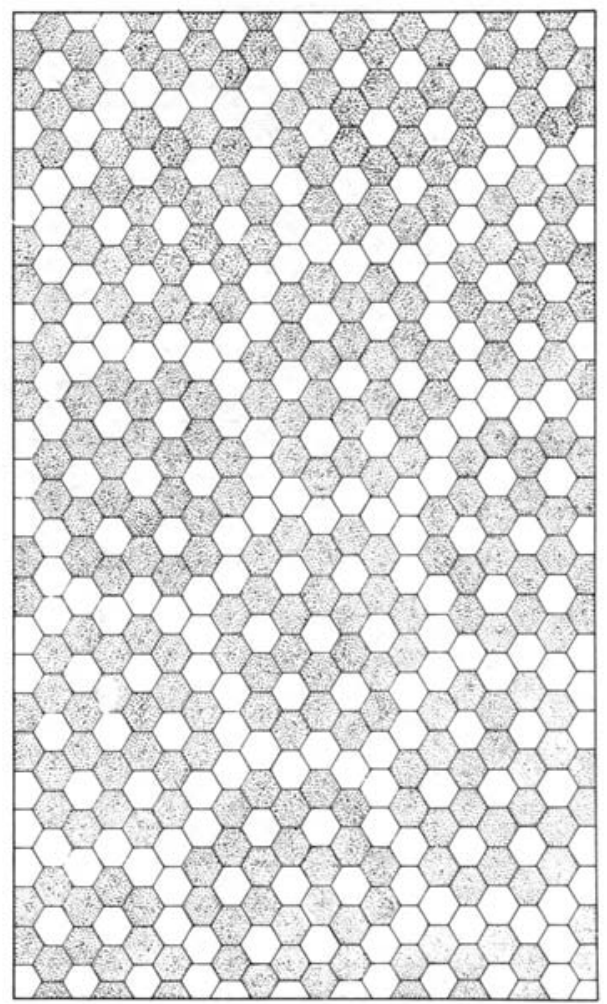

Figure 11. Sequential cogwheel array of period 43.

and have the same periods, $f(n)=4 n^{2}-2 n+1$. The sequence of Figure 15(a) is 0010111110111 (another example with only isolates and 2-strings in one symbol) and for Figure 15(b) is 000101110110111101111 (only 3-strings and isolates in one symbol).

Figure 16 shows another array with three-fold symmetry, of interest in that, provided the marked squares are deleted from the pattern, the white and dotted hexagons form identical motifs. In this particular case, the motif is the symbol of the Isle of Man, but other motifs are also possible in arrays with this property.

\section{Unsolved problems}

Besides the question concerning the uniform non-edge-to-edge tilings mentioned at the end of Section 2, various other unsolved problems remain for the hexagonal grid.

(1) Does there exist a family of binary arrays, such that if we took the union of appropriately sized chunks from each array, we would have a balanced collection? 


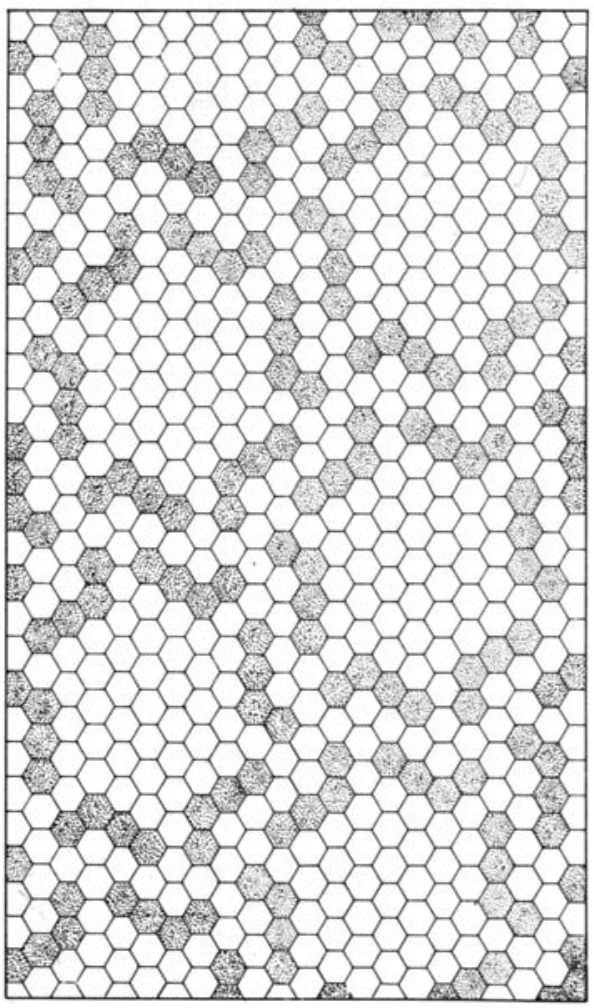

FIGURE 12. Sequential array with six-fold symmetry from asymmetric motif.

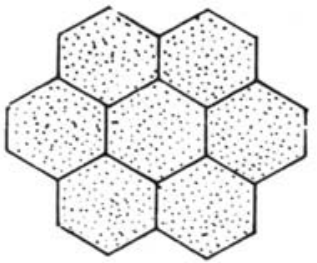

(a)

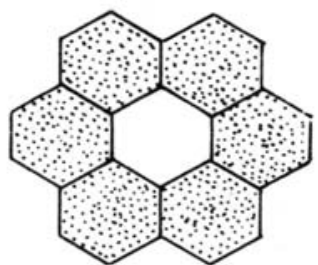

(b)

FIGURE 13. Colouring schemes for the motifs of $P$.

(2) Does there exist a binary array which is balanced if certain rows are not counted, that is, if certain rows are used simply to provide correct neighbourhoods for the remaining hexagons?

(3) How closely may a binary array approximate balance or, in other words, what is the least possible wastage for one plane-filling array?

(4) In each of the above cases where the required array exists, can it be made sequential? 


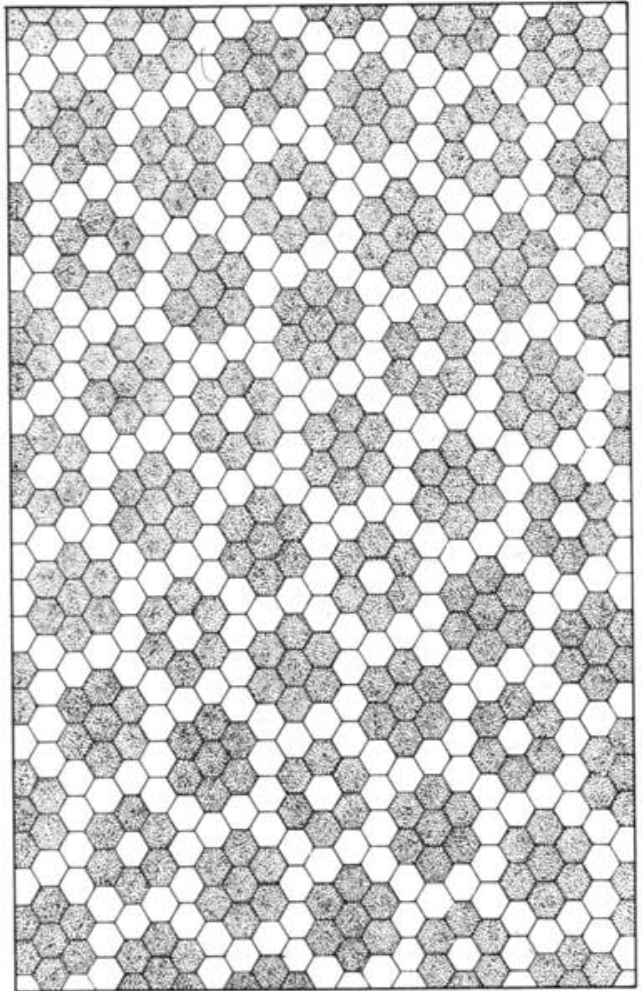

(a)

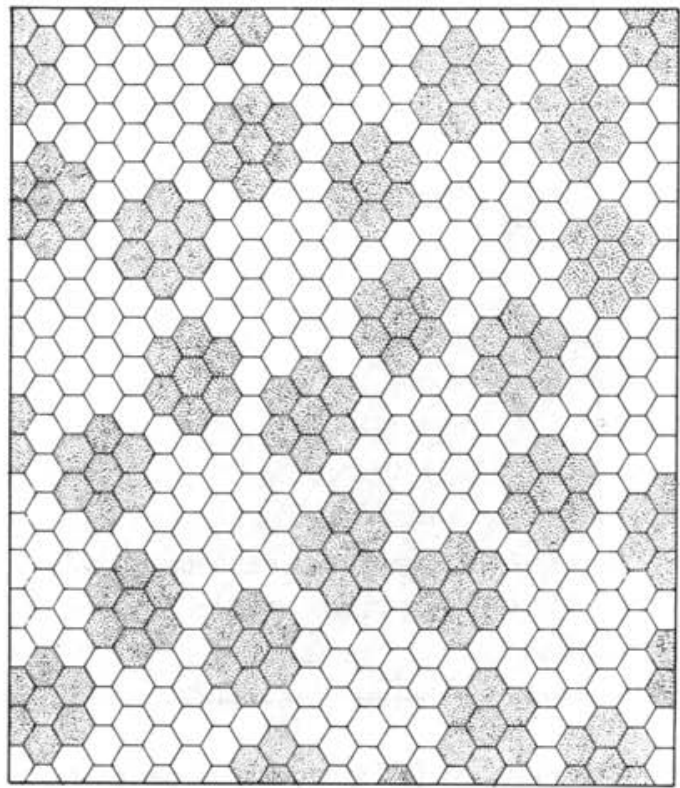

(b)

FIGURE 14. Sequential arrays constructed by composition; (a) period 39, composition $P Q$; (b) period 39, composition $Q P$. 


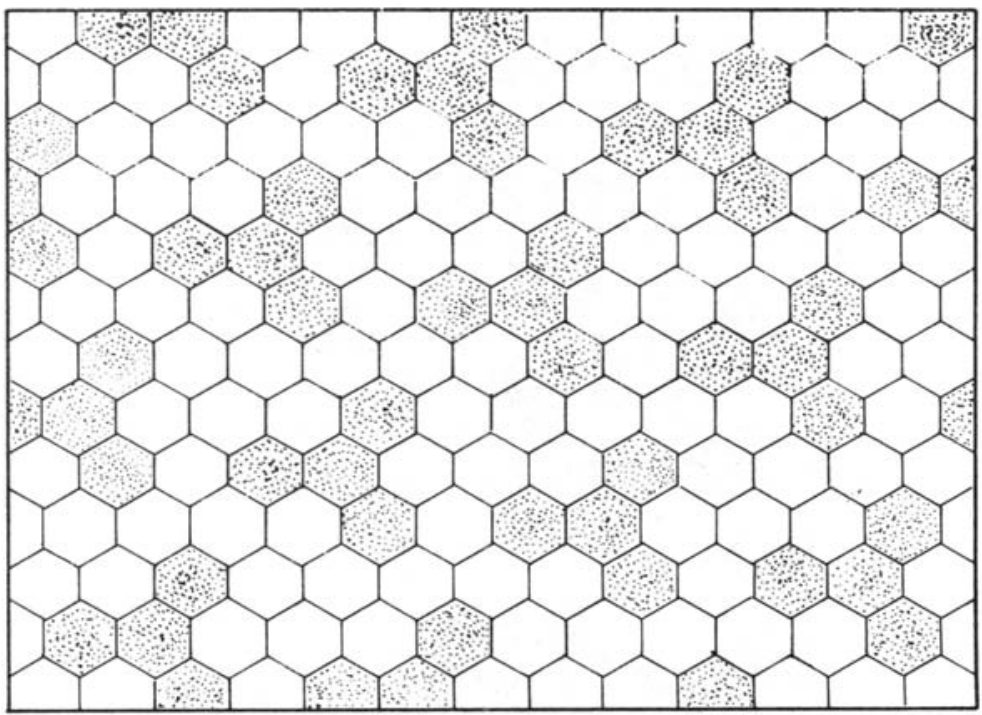

(a)

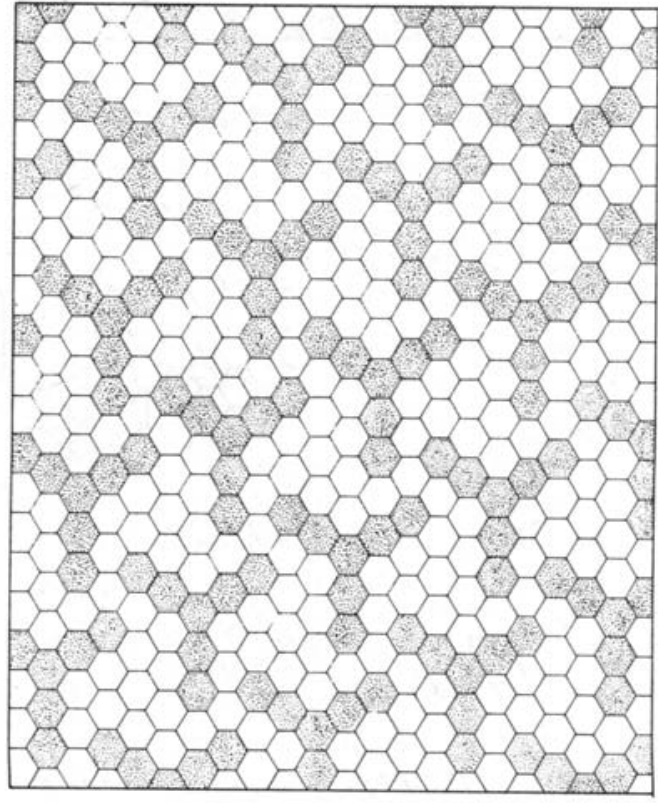

(b)

FloURE 15. Sequential arrays with three-fold synimetry and period $f(n)=4 n^{2}-2 n+1$ : (a) period 13, $n=2$; (b) period $31, n=3$. 


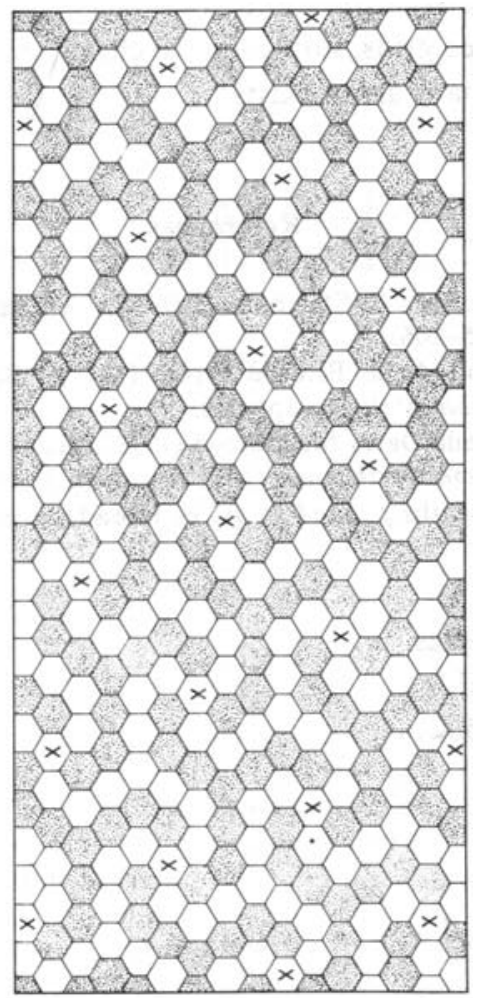

Figure 16. Isle-of-Man array.

(5) Does there exist a balanced ternary array? Certainly the sequence ... 012012 ... gives a sequential array, like a tricolour checkerboard.

(6) All the sequential arrays discussed in Section 3 have odd period, and we conjecture that no sequence of even period exists. If so, then such an array cannot even have equal proportions of the two symbols. Our only inkling of how to attack this problem is as follows.

Suppose that in the array of Figure 9(a), we mark corresponding small hexagons in each hexagonal motif, say the top left hexagon in each. In the horizontal sequence, we have 1101110110000 , with the marked 1 indicated. In the eleven o'clock direction, the sequence becomes 1101110110000 and in the one o'clock 1101110110000 , so that different 1 s are marked in each sequence. However, if we retain these 1 s only, and consider the other twelve elements of the sequence to be 0 , we obtain a sequential array, complementary to Figure 8 . Could this procedure be adapted to prove that if a sequential array of even period exists, then one with sequence $100 \ldots 0$ must exist? This would reduce the problem to dealing with this

$$
\underbrace{}_{2 n-1}
$$

particular type of sequence. 
Finally, the most important question:

(7) For any of the three grids, how may we characterize an equivalence relation for which a balanced binary array exists?

\section{References}

Branko Grünbaüm and Geoffrey C. Shephard (1977-8), 'Tilings by regular polygons', Math. Mag. 50, 227-247; 51, 205-206.

Sheila Oates Macdonald and Anne Penfold Street (1979), 'Balanced binary arrays II: the triangular grid', Ars Combinatoria (to appear).

Anne Penfold Street and Sheila Oates Macdonald (1979), 'Balanced binary arrays I: the square grid', Comb. Maths. VI, Proc. Sixth Aust. Conf. on Comb. Maths (Armidale, 1978) (SpringerVerlag Lecture Notes in Maths., edited by A. F. Horadam and W. D. Wallis) (to appear).

Department of Mathematics

University of Queensland

St. Lucia, Queensland 4067

Australia 